\title{
Celiac Disease Associated with Facioscapulohumeral Muscular Dystrophy
}

\author{
Dorottya Kocsis*, László Herszényi, Miklós Tóth, Zsolt Tulassay, Márk Juhász \\ Semmelweis University, 2nd Department of Internal Medicine, Budapest, Hungary \\ *Corresponding author: kocsisdorottya1@gmail.com
}

Received October 11, 2015; Revised November 09, 2015; Accepted November 12, 2015

\begin{abstract}
Introduction. The association of celiac disease and facioscapulohumeral muscular dystrophy was not reported earlier in the literature. Case presentation. A 35-year-old woman diagnosed clinically with facioscapulohumeral muscular dystrophy 23 years ago. In the family history patients' mother was diagnosed with facioscapulohumeral muscular dystrophy, too. Physical examination revealed bilateral facial weakness, she had narrow smile and murmuring speech. She had low-degree atrophy of shoulder girdle muscles with left-dominated scapular winging. facioscapulohumeral muscular dystrophy DNA testing showed allele 1 deletion. From 2001 on, patient presented epigastrial pain, diarrhoea, weight loss. She had permanent fatigue and she was unable to do regular physical exercises, which led to the progression of muscular dystrophy. Since serological examination detected elevated tissue transglutaminase and deaminated gliadin peptide antibodies levels, and duodenal biopsy revealed subtotal villous atrophy. Celiac disease was diagnosed. She started strict gluten free diet. After 3 months of gluten free diet, patient reported the elimination of gastrointestinal symptoms, better general condition, weight gain and increased muscle strength. Conclusion. Appropriate nutrition is essential for facioscapulohumeral muscular dystrophy patients, therefore detection and treatment of any disorders leading to malabsorption, most commonly celiac disease, are of utmost importance.
\end{abstract}

Keywords: celiac disease, malabsorption, facioscapulohumeral muscular dystrophy, gluten free

Cite This Article: Dorottya Kocsis, László Herszényi, Miklós Tóth, Zsolt Tulassay, and Márk Juhász, "Celiac Disease Associated with Facioscapulohumeral Muscular Dystrophy." International Journal of Celiac Disease, vol. 3, no. 4 (2015): 162-164. doi: 10.12691/ijcd-3-4-9.

\section{Introduction}

Celiac disease (CeD) is a widespread autoimmune disorder that is initiated by the ingestion of wheat gluten and other proteins related to rye and barley in genetically predisposed individuals, characterized by the presence of a variable combination of gluten dependent clinical manifestations, $\mathrm{CeD}$ specific antibodies, HLA-DQ2 and DQ8 haplotypes and enteropathy. However, CeD may manifest itself at any age, with the potential involvement of any organs. In adulthood, "classic symptoms" including diarrhea, abdominal distension, malabsorption syndrome as typical clinical feature are commonly absent. Patients may exhibit minor gastrointestinal complaints, as well as numerous extraintestinal manifestations. The prevalence of $\mathrm{CeD}$ is roughly $1 \%$. For the diagnosis of $\mathrm{CeD}$ in dietnaive adult patients serological and histological examination is necessary. Currently, the only appropriate treatment for $\mathrm{CeD}$ is a lifelong gluten free diet (GFD) [1].

Nowadays, the range of diseases that can be proven to occur more frequently in untreated $\mathrm{CeD}$ has expanded. $\mathrm{CeD}$ or gluten sensitivity may initially present as one or more neurological signs and/or symptoms. On the other hand, it may be associated with or complicated by neurological manifestations. Neurological manifestations can be seen in nearly $10 \%-36 \%$ of CeD patients, the most common being cerebellar ataxia and neuropathy. [2,3] Other neurological manifestations are epilepsy, cognitive disorders, dementia, tremor, myelopathy, neuropathy, brainstem encephalitis, progressive leukoencephalopathy, vasculitis, occipital calcification, anxiety/depression, and myoclonic syndrome. They also include neuromuscular manifestation such as peripheral polyneuropathy, mononeuropathy multiplex, dermatomyositis, polymyositis, and inclusion body myositis [4].

Facioscapulohumeral muscular dystrophy (FSHD) is a common type of adult muscular dystrophy and is divided into types 1 and 2 based on genetic mutation. [5] Clinically, in both FSHD types patients suffer from a progressive and irreversible weakness of the facial, shoulder and upper arm muscles. With disease progression, other muscles may also become affected. Interestingly, muscle weakness in FSHD is often asymmetric. Symptomatic non-muscular disease manifestations are rare but can include sensorineural deafness, retinovasculopathy and intellectual disability. Pain and fatigue is a frequent complaint. [6] Approximately 95\% of patients, termed FSHD1, have a deletion of a key number of repetitive elements on chromosome $4 \mathrm{q} 35$. The remaining 5\%, termed FSHD2, have no deletion on chromosome 4q35. With an incidence between 1:15,000 and 1:20,000 FSHD is the third most common myopathy. $[7,8]$ The age at disease onset ranges from infancy to 
middle age with the majority becoming symptomatic in the second and third decade of life. [9] Nowadays, no therapy is available for FSHD. However, patients usually report some improvement related to physical exercise and the use of nutritional supplements $[10,11]$.

The aim of this case report is to show an unusual association between $\mathrm{CeD}$ and FSHD.

\section{Case Report}

A 35-year-old woman diagnosed clinically with facioscapulohumeral muscular dystrophy 23 years ago by pediatrician, with left side scapula alata being the most prominent symptom. Family history revealed a strong maternal familial inheritance pattern. Mater diagnosed with atypical muscle dystrophy, when she was twentyfour years old, based on weakness of lower limb muscles, incomplett foot dorsalflexion and waddling gait. Neurological examinations were conducted at Department of Neurology, Heim Pál Children's Hospital, Budapest, Hungary. Physical exam revealed bilateral facial weakness, most prominent on her mouth, resulting in narrow smile and murmuring speech. She had low-degree atrophy of shoulder girdle muscles with left-dominated scapular winging. Electrophysiological studies revealed peripheral neurogenic lesions in left side supraspinatus muscle and bilateral serratus anterior muscles, and severe myogen lesion in orbicularis oris muscle. Lower limbs muscles were not affected. At the time of her diagnosis, creatine phosphokinase (CPK) level was elevated. These results confirmed the diagnosis of FSHD. The initial management of FSHD was to take vitamin E, and to do regular physical exercises. During follow-up, her general condition were unchanged until 2001, when she neglected physical exercises for one month. After that, she had weakness in her proximal hip muscles, gluteus medius muscle, and difficulties with walking. Because of her continuously increasing lumbar lordosis it became necessary to wear a corset temporary. She received creatine monohydrate terapy, without any significant improvement. In 2004, DNA test were performed by patient and her mother: both FSHD tests showed allele 1 deletion. From 2001 patient presented gastrointestinal symptoms, like epigastrial pain, diarrhoea, and weight loss. Gastroscopy was performed revealing gastrooesophageal reflux disease; at that time, duodenal biopsy was not taken. She was treated with protonpump-inhibitor (lanzoprazol). Because of recurrent abdominal discomfort and temporary occurring diarrhea, colonoscopy was performed in 2012, with negative result. Patient then went on to suffer from chronic diarrhea, weight loss and permanent fatigue and she was unable to do regular physical exercises. Patients BMI (body mass index) was $18,7 \mathrm{~m} 2 / \mathrm{kg}$. Consequently, her muscular dystrophy progressed. Suspicion of malabsorption, CeD in particular, was raised. Serology proved high levels of IgA tissue transglutaminase antibody (tTG IgA:292 IU, and tTG IgG: 2 IU, respectively) and IgA and IgG deamidated gliadin peptide antibodies (DGP IgA:228 IU, and DGP IgG:100, respectively). Duodenal biopsy revealed subtotal villous atrophy crypt elongation, increased intraepithelial lymphocyte / epithelial cell ratio, providing a Marsh 3b lesion and thereby the clinical diagnosis of CeD (Figure 1). Somewhat surprisingly, DEXA-based osteodensitometry has shown normal bone mineral density. Patient started a strict GFD, and was provided with nutritional supplements. After 3 months of GFD, patient reported eliminated gastrointestinal symptoms, better general condition, weight gain and increased muscle strength her BMI value was $19,89 \mathrm{~m} 2 / \mathrm{kg}$. She continued with physical exercises.

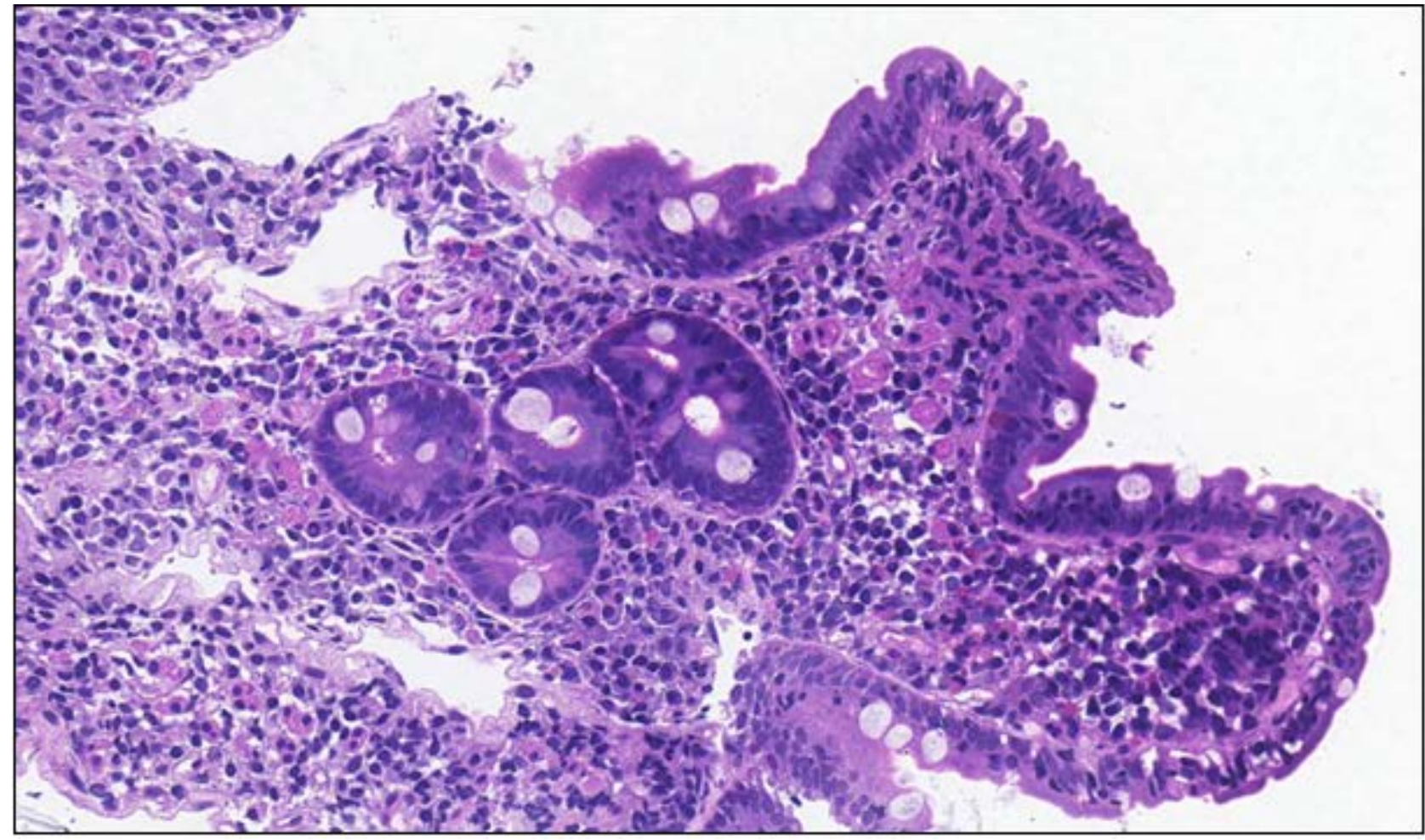

Figure 1. Histological picture paint with hematoxilin-eosin. Duodenal biopsy revealed subtotal villous atrophy crypt elongation, increased intraepithelial lymphocyte / epithelial cell ratio, providing a Marsh 3b lesion 


\section{Discussion}

The association of $\mathrm{CeD}$ and FSHD was not reported earlier in the literature. The co-appearance of two diseases could be coincidental, but nowadays many evidence available to suggest $\mathrm{CeD}$ can present neurological symptoms or be associated with neurological or neuromuscular disorders.

The pathomechanism underlying of the neurological manifestation of $\mathrm{CeD}$ is still unknown. Recently, there are several hypotheses about gluten toxic damage and vitamin malabsorption. [4,5] Neuropathy in CeD patients presumably mediated in part by antiganglioside antibodies or by antibodies that target transglutaminase bound to extracellular proteins such as fibronectin. However, these mechanisms have not yet been established $[12,13]$.

Numerous studies have reported neurological manifestations in $\mathrm{CeD}$ that shown improvement following the administraiton of GFD [13,14].

FSHD is the third most common muscular dystrophy, with a prevalence of 1/20,000. Up until now, no curative treatment is available. Corticosteroids, albuterol, creatine monohydrate and myostatin have not been beneficial. Physical exercise, and nutritional supplements (vitamin E, B12, B6, D etc.) improve strength and endurance, and may slow the progression of muscular dystrophy. [11] In $\mathrm{CeD}$, villus atrophy inhibit the absorption of these nutritional supplements, and generalized fatigue and weakness may also occur caused by malabsorption. In this reported case, introduction of GFD resulted in not only the elimination of gastrointestinal symptoms, but also in the improvement of symptoms related to muscular dystrophy.

\section{The Take Home Messages for the Clinicians}

Appropriate nutrition is essential for patients with FSHD; disorders leading to malabsorption contribute to the deterioration of neurological status, therefore alertness towards these gastrointestinal diseases, most commonly $\mathrm{CeD}$, is vital.

\section{References}

[1] Husby S, Koletzko S, Korponay-Szabo IR, et al. European Society for Pediatric Gastroenterology, Hepatology, and Nutrition guidelines for the diagnosis of celiac disease. $\mathrm{J}$ Pediatr Gastroenterol Nutr. 2012; 54: 136-160.

[2] Chin RL, Sander HW, Brannagan TH, et al. Celiac neuropathy. Neurology. 2003; 60: 1581-1585.

[3] Hadjivassiliou M, Grünewald RA, Chattopadhyay AK, et al. Clinical, radiological, neurophysiological, and neuropathological characteristics of gluten ataxia. Lancet. 1998; 352: 1582-1585.

[4] Chaudhry V, Ravich WJ. Neurology and General Medicine. 3th ed. New York, NY: Churchill Livingstone; 2001. Other neurological disorders associated with gastrointestinal, liver, or pancreatic diseases; pp. 283-4.

[5] Tawil R, Van Der Maarel SM, Tapscott SJ. Facioscapulohumera dystrophy: the path to consensus on pathophysiology. Skelet Muscle. 2014; 4: 12

[6] Statland JM, Tawil R. Facioscapulohumeral muscular dystrophy: molecular pathological advances and future directions. Curr Opin Neurol. 2011;24: 423-428.

[7] Tawil R, Van Der Maarel SM. Facioscapulohumeral muscular dystrophy. Muscle Nerve. 2006; 34: 1-15.

[8] Mostacciuolo ML, Pastorello E, Vazza G, et al Facioscapulohumeral muscular dystrophy: epidemiological and molecular study in a north-east Italian population sample. Clin Genet. 2009; 75: 550-555.

[9] Lunt PW, Compston DA, Harper PS. Estimation of age dependent penetrance in facioscapulohumeral muscular dystrophy by minimising ascertainment bias. J Med Genet. 1989; 26: 755-760.

[10] Olsen DB, Orngreen MC, Vissing J. Aerobic training improves exercise performance in facioscapulohumeral muscular dystrophy. Neurology 2005, 64: 1064-1066.

[11] Pasotti S, Magnani B, Longa E, et al An integrated approach in a case of facioscapulohumeral dystrophy BMC Musculoskeletal Disorders 2014, 15: 15.

[12] Chin RL, Sander HW, Brannagan TH, et al. Celiac neuropathy. Neurology. 2003; 60: 1581-1585.

[13] Alaedini A, Green PH, Sander HW, et al. Ganglioside reactive antibodies in the neuropathy associated with celiac disease. J Neuroimmunol. 2002; 127: 145-148.

[14] Akimov SS, Krylov D, Fleischman LF, et al. Tissue transglutaminase is an integrin-binding adhesion coreceptor for fibronectin. J Cell Biol. 2000; 148: 825-838. 